\title{
Exploration of Object-Oriented Models in Informatics Education
}

\author{
Torsten Brinda and Sigrid E. Schubert \\ Didactics of Informatics, University of Dortmund, D-44221 Dortmund, Germany
}

\{brinda,schubert\}@ls12.cs.uni-dortmund.de

Key words: Basic Concepts, Didactics of Informatics, e-learning, Learner-CentredLearning, Learning Environments

\begin{abstract}
The authors introduce the exploration of object-oriented basic concepts as a new approach to object-oriented modelling (OOM). While other subjects have a long tradition in learning by discovery with suitable learning aids (e.g. experimental installations of the scientific subjects), Informatics at first has to define and to develop such learning aids. We therefore analysed software development environments and identified the factors that promote understanding and those that inhibit understanding. In particular we found that "support of the syntactic correctness of a model", "promotion of the logical correctness of a model" and "visualisation of abstract concepts with change of the observation perspective" were factors that promoted understanding. Understanding, however, was hindered or inhibited by factors such as "error avoidance by ban of action" as well as the fact that the fundamental ideas of OOM are presupposed. From these findings, design criteria of the Didactics of Informatics for so-called "exploration modules" which are learning aids for the exploration of object-oriented basic concepts, were derived. These key criteria are the "basic concepts on different abstraction levels" and the "synchronisation, transformation and evaluation of diagrams". Finally the role of exploration modules as a lesson medium is discussed and the application of the exploration by learners is explained. The change between different views of an object-oriented model to ensure its completeness and consistency is stressed.
\end{abstract}

The original version of this chapter was revised: The copyright line was incorrect. This has been corrected. The Erratum to this chapter is available at DOI: 10.1007/978-0-387-35663-1_34 


\section{MOTIVATION FOR EXPLORATION}

Education has to prepare learners for the changing everyday world. Due to their importance in society, the study of informatics systems (particularly computer networks and distributed systems) and object-oriented modelling (as a systematic method of informatics system construction and analysis), are essential components of informatics education.

In the past small programs were developed for informatics education to help learners derive knowledge about real world applications. This worked well as long as both lesson examples and professional solutions were based on the same principles and methods. Learners followed the same strategies as experts, though with objects of greatly reduced complexity. Meanwhile, the algorithms, data structures and software development methods on which large informatics systems are based have changed. Consequently the transfer from 'programming in the small' (simple examples for algorithms and data structures) to 'programming in the large' (principles of software engineering by means of complex examples) is no longer recommended (Applerath et al 1998).

The activities of learners in informatics lessons are frequently limited to the application of standard software and the exploration of problem solving with the help of a programming language. As educational objectives have changed object-oriented programming languages have become an important focus of study. The modelling language 'Unified Modelling Language (UML)' and related tools for analysis and design are increasingly used in informatics lessons. These tools are, however, considered to be less suitable for informatics modelling in informatics education, because the complexity of their user interfaces ties up a lot of lesson time. This is a symptom of the original objective of software development environments (SDEs) - to support professional software developers in producing or constructing efficient and error-free software products (Figure 1).

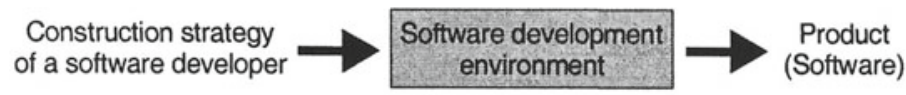

Figure 1. The software development process

SDEs provide functions e.g. 'error avoidance by ban of action' that turn out to be barriers which may be experienced in the educational process of learning. This feature (e.g. selection of the return type of a method from a list of data types) is very useful for the avoidance of certain error classes, but critical if permanently activated from a didactic viewpoint, because then learners are protected from certain error classes and learning by error cannot 
occur. When working without a SDE or with a SDE that does not offer such functionality, learners become confronted with such errors without having learned corresponding problem solving strategies. In view of the educational process the main problem is that the fundamental ideas of the object of consideration (here: the construction of object-oriented models) are presupposed. These systems address users with pre-knowledge in the design of object-oriented problem solutions and help them to take into account everything essential for modelling, to automate routine tasks and to increase the quality of their solutions. With respect to the teaching and learning of modelling strategies these systems have not been developed, nor do we feel they would be suitable for this task.

Product development and the education of software developers are not objectives of informatics education. Modern concepts for informatics education at secondary and university level stress the active role of the learners to prepare them adequately for self-paced, self-organized and lifelong learning. Learning and teaching concepts that should feature more in education are active, collaborative, peer and explorative learning (Turner 1998). Learning aids to support learners in their educational process are essential (Figure 2).

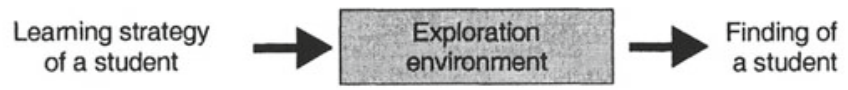

Figure 2. The exploration process

Therefore we developed 'Didactic Systems' as a combination of traditional and modern study concepts. The didactic system for objectoriented modelling makes it possible for learners to navigate in knowledge structures, to construct solutions for exercises from 'Exercise Classes' and to learn by discovery with exploration modules (EMs) (Brinda and Schubert 2001). All these learning forms are embedded in the social processes of the informatics lesson.

\section{EXPLORATION WITH SOFTWARE MODULES}

The exploration process is extremely important and it is valuable to consider the learning aids that it would be desirable to develop for Informatics. The scientific subjects make extensive use of experimental installations and technology education has a long tradition of working with construction kits.

The Didactics of Informatics group at the University of Dortmund 
developed a software environment in which learners can construct a part of the Internet architecture in a closed environment and then check the data communication between network components using a protocol simulation (Steinkamp 1999). Learners work directly with a 'construction sheet' window in which they construct and connect components. The data input and output of each component is shown on another window - the 'communication monitor'.

Learners can also see if data communication was not possible due to incorrect connection of components. Learners easily recognize whether or not their construction is correct because the requested web document, or a message to say that this document is not available, appears in a simulated browser window. Through use of this learning aid, it is expected that understanding of the working principles of distributed informatics systems is improved. We intend to develop EMs which we will use in teaching about object-oriented modelling.

\section{UNDERSTANDING PROMOTING FUNCTIONS OF SOFTWARE DEVELOPMENT ENVIRIONMENTS}

Through the analysis of SDEs, especially modelling tools, the following functions which promote understanding have been identified. These functions are essential for the design of exploration modules.

\subsection{Support of the syntactic correctness of a model}

Modelling tools provide correct or validated model components as predefined building blocks. The user selects from these correct or validated alternatives. The syntactic correctness of the model remains unchanged as long as a valid combination is chosen. Source code generators are attached to many modelling tools with the related compilers. Successful compilation requires a syntactically correct model. Reports from the system help the learners to identify any syntactic weaknesses in their models. The understanding process is based on, and develops from, the discussion processes within the group of learners.

\subsection{Promotion of the logical correctness of a model}

Every modelling language is based on a grammar, which defines how the single building blocks may be connected with each other. For example, in a 
class diagram e.g. inheritance cycles are not permitted. The user is informed when construction rules are disregarded. Typical classes of logical errors are avoided. The assurance of the completeness and consistency of models also belongs to this field. Certain models, like class diagrams, allow the user to model quite informally at first. This is highly desirable in early phases of the modelling process.

Turning to more formal representations, abstract concepts like a class constructor, the formal specification of earlier informally defined attributes and methods and the access operations for the attributes are indispensable. In many cases a software development tool can identify such missing details and ask the user to complete the model. In addition such tools can ensure the consistency of different sub-models (e.g. class and sequence diagrams). Through this process learners understand which components belong to a complete model and how the consistency of different sub-models can be realized. Deeper problems must be identified and resolved in lesson discussions. It would be naive to count on system support to offer solutions to the tremendous variety of potential semantic (logical) problems.

\subsection{Visualization of abstract concepts with change of the observation perspective}

Modelling tools offer the possibility to change the size of diagrams and the degree of detail. Learners can view the information at or on different levels of abstraction. They can concentrate on the elements that are essential for a concrete problem solution phase. In order to, e.g. analyse the structure of a class hierarchy, the representation of detailed information on single classes (attributes and functions) is not required if inheritance is not the topic of discussion. SDEs offer multiple step execution and undoing of functions. Of course learners expect such application standards in software that is needed for exploration. Since many of the afore-mentioned model check mechanisms only indicate problems but are unable to correct them automatically, learners soon become aware of their own errors. It remains a complicated learning process to understand why an error occurred (Tholander 2001) and even more difficult to derive better strategies for future modelling tasks from understanding the error. The traditional educational process allows for the implementation of correction recommendations worked out by the learning group. 


\section{DESIGN CRITERIA OF THE DIDACTICS OF INFORMATICS}

The concept of exploration is a component of the educational process. Therefore learning theories and the principles and methods of the Didactics of Informatics crucially influence the arrangement of that concept. The learning theory of constructivism (Vygotsky 1978), which is eminently suitable for accommodating various constructive requests of informatics education, is applied. From our examination of the didactics of informatics we have concluded that learners in the context of OOM (Rumbaugh et al 1991) should:

- $\quad$ get to know and design structures (static basic concepts);

- understand and control processes (dynamic basic concepts);

- analyse, modify, construct and assess object-oriented models following the procedure of select elements, structure, model in developing examples of use of the everyday world.

Therefore the authors consider the following components and functions of courseware promote exploration.

\subsection{Basic concepts on different abstraction levels}

In order to give learners with varied pre-knowledge a cognitively demanding but not overtaxing entry to object-oriented modelling, the basic concepts must be capable of exploration at different levels of abstraction. For beginners, representation forms are suitable which should illustrate the basic idea of object-orientation in examples of the everyday world. An animation view on a reality section shows the objects, their attributes and behaviour. Advanced learners are introduced step by step to more formal representation forms such as selected UML diagrams. The meaning and effect of object view, class view, and message exchange view must be explored.

At least one static and one dynamic representation form are necessary to describe the architecture and the course (the time change of the state of an object network). The class diagram as a central structure description of the architecture of object-oriented models is inalienable. To illustrate the dynamics of the message exchange, sequence and collaboration diagrams that emphasise various aspects of the process are invaluable. While methods which exclusively reach their behaviour by communication with other objects can be modelled superbly by means of sequence diagrams, calculation rich methods which have low, or no, object communication can only be partially designed with their aid. For this reason structograms are used within imperative problem solving and a suitable combination of 
sequence diagrams and structograms must be developed for an exploration. The step from a reality section to a class diagram can be simplified with object diagrams.

It must be possible to switch selected diagrams on or off. The complexity of the diagrams is influenced by the fact that components and details may be hidden or uncovered. In an OOM various processes run, objects become instantiated, links to other objects are built up and removed, messages between objects are sent, objects are destroyed. To be able to understand these processes and to judge their consequences the processes are activated by the learners, watched, recorded and discussed. On a graphic level, independently of a special programming language, objects of a class of a given class structure are created, their methods executed and the changes in the object states monitored.

\subsection{Synchronization, transformation and evaluation of diagrams}

Synchronization represents a 'didactic bridge' between different diagrams. This makes it possible to bring the single diagrams together to form a picture of the complete system and thus overcome a known cognitive barrier. These may be a set of diagrams that represent the same information under different points of view. As far as it is possible, these views must be synchronized automatically. An example is the representation of a simple reality section, e.g. the move operation on a graphic object, its representation within an animated collaboration diagram and the synchronized representation of the corresponding source code. In addition there is the possibility of recording running processes in the system and rerunning the recording later in different views. It is not always possible or desirable that the exploration system automatically synchronizes views. In order to enable learners to transform representation forms on their own e.g. the transformation of class to object diagrams and vice versa, functions must be provided with which the consistency of views can be checked. For every object diagram a well-defined class diagram can be determined but the reverse is impossible, because an unlimited number of object diagrams can be derived from a class diagram. However, we can check whether an object diagram is a valid instance of a class diagram and we can identify where the structure is damaged. 


\section{EXPLORATION MODULES AS LESSON MEDIUM}

To embed the concept of exploration in the traditional educational process we need to develop suitable learning aids (EMs). With this software collection learners can find out connections and discuss their discoveries during the lesson with the learning group. Interactions between the learners and the EMs are very important. We haven't used learning texts as part of the EMs but good textbooks on OOM are available during the lesson.

EMs have a structure like the technical experimenting box of building blocks. Learners combine solution elements to object-oriented models. They can examine predefined models or design their own models. The diagnosis options of models from the database are extensive. For freely chosen designs a standard set of representations can be used for illustration and exploration. However, an extensive model critique is carried out in the lesson conversation. EMs were developed for learning object-oriented basic concepts and they complement the use of software development tools as a learning aid. The pressure on learners from the syntax and semantics of a programming language in the first lessons of the course is avoided by the use of EMs (Schubert 2000).

To examine a learning object with an EM suitable observation and manipulation components are required. Complex connections, principles and methods are illustrated and exemplified. The observation component manages all diagrams (views), animations and the changing of views. It also is used to generate sub-models automatically (e.g. class diagram to a given object diagram). EMs stimulate learners to develop hypothesis formulation about the object of examination giving them the opportunity to explore the object, manipulate it and check whether changes cause expected consequences. This is possible with all construction and parameterisation processes of object-oriented models. In addition, at the half-automated view transfer (e.g. from class diagram to object diagram or from class diagram to real object) learners must interact with the system and make decisions.

In this context the dialogue component of the EMs is extremely important. It presents learners with the consequences of their actions. In the learning process the two main areas of observation and manipulation are combined. An object of examination is altered and carefully observed. The consequences of the actions are analysed and judged. Following a modified plan observation and manipulation are continued. This process leads to a higher level of understanding. 


\section{LEARNERS' APPLICATION OF EXPLORATION}

Exploration promotes learning-by-doing oriented, problem and situation based learning when correctly integrated in the informatics education (van Weert 2001) The recommendation of learning scenarios illustrates this (Table 1, number of points as indicator for the degree, how much the authors recommend the application in the informatics education).

In object oriented modelling the linking of basic concepts with model elements, selected views of a model and the view change (respectively the combination of different model views) requires an explorative approach. In all cases the abstract connections are processed in a way that makes interaction with the concepts possible. The observations of the learners are embedded in the theory of object-oriented modelling in the informatics lesson and therewith turn to knowledge.

Table 1. Application of exploration in Informatics education

\begin{tabular}{|c|c|c|c|c|}
\hline Lesson phases & $\begin{array}{c}\text { Model } \\
\text { elements }\end{array}$ & Model view & View change & $\begin{array}{c}\text { Informatics } \\
\text { system }\end{array}$ \\
\hline Problem orientation & ....... & .... & $\cdots$ & .• \\
\hline Motivation & & .... & ...... & \\
\hline $\begin{array}{l}\text { Introduction to new } \\
\text { material }\end{array}$ & .• & .. & .. & .• \\
\hline Exercise/application & .. & .. & .• & .. \\
\hline Subject specific transfer & & .. & ...... & \\
\hline $\begin{array}{l}\text { Controls of learning } \\
\text { success }\end{array}$ & & & .. & \\
\hline
\end{tabular}

However, exploration is not equally effective in all areas of OOM and all the afore-mentioned lesson phases. We must have realistic expectations of exploration. Our research shows that exploration can be particularly recommended for problem orientation in the fields of model elements, model view and view change. Using specific visualisation and interaction possibilities this is especially valid for single model elements. In the lesson phases concerned with motivation and subject specific transfer, the view change of the learners is promoted due to computer supported synchronisation of views and their visualisation. When learners discover the meaning of a view change in an example world they are motivated to work with this method (Table 1).

The EMs have a double pedagogical function, because they can either be used as learning aids or can be analysed and discussed as an example of a complex software system. The discovery progress of the learners is tied to general conditions e.g. the establishment of quality demands as well as 
inclusion in teacher education. Student teachers of informatics on the one hand have to be prepared to use exploration as a new teaching method and on the other hand to see EMs as learning aids.

In the process of the development of the 'Didactic System for OOM' the following steps are the next to be explored. The activities of learners and concrete examples for lesson phases with EMs will be worked out. A group of 11 students of Informatics at the University of Dortmund will implement an exploration environment for selected object-oriented basic concepts until July 2002 and the component 'Exercise Classes' (Brinda and Schubert 2001) of the didactic system will be applied and empirically evaluated in student teacher education of informatics in spring 2002.

\section{REFERENCES}

Appelrath, H.-J., Boles, D., Claus, V. and Wegener, I. (1998) Starthilfe Informatik. B.G. Teubner, Stuttgart.

Brinda, T. and Schubert, S. (2001) Didactic system for object-oriented modelling. In Networking the Learner, Proceedings of the seventh World Conference on Computers in Education (WCCE 2001), Kluwer Academic Publishers, Boston, pp 473-482.

Rumbaugh, J., Blaha, M., Premerlani, W., Eddy, F. and Lorensen, W. (1991) Object-Oriented Modelling and Design. Prentice-Hall, New York.

Schubert, S. (2000) The impact of modelling in informatics education on collaborative learning with school Intranets. In The Bookmark to the School of the Future, P. Hogenbirk and H. G. Taylor, (eds.), Kluwer Academic Publishers, Boston, pp. 247-258.

Steinkamp, D. (1999) Informatik-Experimente im Schullabor. Diploma thesis, Dept. of Informatics, University of Dortmund.

[http://ddi.cs.unidortmund.de/lehre/diplom/steinkamp/]

Tholander, J. (2000) Students Interacting through a Cognitive Apprenticeship Learning Environment. Euro-CSCL 2001, Maastricht.

[ http://www.mmi.unimaas.nl/euro-cscl/Papers/162.pdf]

Turner, A. J. (1998) Trends in teaching informatics. In Informatics in Higher Education, F. Mulder and T. van Weert, (eds.), Chapman and Hall, London.

van Weert, T. (2001) Co-operative ICT-supported learning. A practical approach to design. In: Informatikunterricht und Medienbildung, R. Keil-Slawik and J. Magenheim (eds.), Köllen, Bonn, pp. 47-62.

Vygotsky, L. S. (1978) Mind in Society. Harvard University Press, London. 